\title{
Aptamers: Promising Molecules for Cancer Stem Cell Targeting
}

\author{
Said I Ismail ${ }^{1 *}$, Walhan Alshaer ${ }^{2}$, Nidaa Ababneh ${ }^{1}$ and Elias Fattal ${ }^{2}$
}

${ }^{1}$ Molecular Biology Research Laboratory, Faculty of Medicine, University of Jordan, Amman 11942, Jordan

${ }^{2}$ Université Paris-Sud, CNRS UMR 8612, Faculté de Pharmacie, 5 Rue Jean-Baptiste Clément, Châtenay-Malabry, France

\begin{abstract}
The term Cancer Stem Cells (CSCs) has been coined to refer to a subpopulation of tumor cells that has the ability to self-renew and to generate the diverse cell pool of a given tumor. In the last decade CSCs have been receiving a lot of interest due to their cancer initiating and maintaining capabilities making them the real driving force within a malignant mass that pushes towards more aggressive proliferation and more resistance against anticancer drugs. These cells have been linked to different specific markers in an attempt to achieve efficient isolation and characterization. Such markers include CD44, EpCAM and CD133 among others.
\end{abstract}

Aptamers are synthetic single stranded oligonucleotides selected from a huge pool of random sequences which can fold and bind to a wide range of targets with high affinity and specificity. They are not immunogenic or toxic and have good clearance rates, qualities that make aptamers a good rival to monoclonal antibodies in their diagnostic as well as therapeutic applications.

This review explores the potential applications of aptamers selected to target the main markers of CSCs. Such applications include diagnostic assays and analytical platforms where such aptamers can be utilized to detect and characterize the behavior of these cells in addition to predicting and monitoring disease course and response to different treatment regimens. The potential therapeutic applications are also discussed in details, where such antiCSC aptamers can be used in a variety of formulations to efficiently target the initiating cores of tumors.

\section{Keywords: Aptamers; SELEX; Cancer stem cells}

\section{Introduction}

Cancer is one of leading cause of death around the world and accounts for around $13 \%$ of world wide deaths according to the World Health Organization (WHO) [1]. Cancer is a general term describing a large number of disorders that can affect various tissues and organs within the body. One main feature common to all cancers is the continuous and uncontrolled proliferation of cells which usually spread and invade neighboring or even distant tissues in a process known as metastasis. Most current treatment regimens aim at restraining this proliferation and metastasis of such cells. However, most of these regimens encounter some poorly understood clinical events, including drug resistance, minimal residual disease, and tumor relapse [2]. One of the main hypotheses that attempt to explain such events states that malignancies depend on a small population of stem-like cells for tumor initiation and proliferation, where these cells, which are more resistant to therapeutic drugs, constitute the main driving force for cancer maintenance [3]. These cells were therefore given the name "cancer stem cells" (CSCs).

As more evidence supports the CSC hypothesis, better characterization and targeting of such cells could have major impacts on the ongoing battle against cancer [4]. The "blind" chemotherapy treatment for cancer patients with its poor outcome and unbearable side effects will become something of the past once the new generation of "smart" targeted drugs becomes available. Such drugs are usually led to their target cells by antibodies, but recently, a new rival for antibodies is emerging under the name of aptamers. These are single stranded nucleic acid ligands with highly complex three-dimensional structures and recognition properties capable of binding tightly and specifically to targets ranging from small molecules to complex multimeric structures. Several aptamers were evolved to target cell specific biomarkers including those on the surface of tumor cells [5-7]. Such aptamers have been used for targeted delivery of diverse cargos including chemotherapeutic agents, nanoparticles, and drug- encapsulated liposomes. The mechanism of delivery is based on the specific interaction between the aptamer and its membrane receptor, which enhances the internalization and accumulation of therapeutic agents inside the target cells. Such delivery would increase drug potency and limit unwanted toxic side effects [8].

\section{The Concept of Cancer Stem Cells}

Within the context of the evolving hypothesis of CSCs, scientists are stressing the fact the tumor mass is made up of a heterogeneous group of cells. These include, rapidly proliferating cells in addition to post-mitotic differentiated cells, as well as a smaller number of stem cells capable of long term self-renewal. Scientists are carefully differentiating between two key types of tumor cells; the cell-of-origin and the CSC. The cell of origin mostly refers to the cell that received the first genetic alterations in the transformation process, thereby referred to as the tumor initiating cell, while the CSC is the cell that holds the ability to propagate the tumor. Indeed, there is a growing evidence of a phenotypic variation between the two cells. Such variation is in support of what has been largely observed with regards to the heterogeneity of the cellular composition of various tumors in terms of the genetic alterations involved, proliferation potential, cellular phenotypes and therapeutic response $[4,9,10]$.

${ }^{*}$ Corresponding author: Dr. Said I. Ismail, Molecular Biology Research Laboratory, Faculty of Medicine, The University of Jordan, Amman 11942, Jordan, Tel: 0096265355000 ext 23472; E-mail: sismail@ju.edu.jo

Received July 29, 2013; Accepted November 21, 2013; Published November 25, 2013

Citation: Ismail SI, Alshaer W, Ababneh N, Fattal E (2013) Aptamers: Promising Molecules for Cancer Stem Cell Targeting. J Mol Genet Med 7: 90 doi: 10.4172/1747-0862.1000090

Copyright: (C) 2013 Ismail SI, et al. This is an open-access article distributed under the terms of the Creative Commons Attribution License, which permits unrestricted use, distribution, and reproduction in any medium, provided the original author and source are credited 
There are two main mechanisms that attempt to explain tumor development and heterogeneity. The first refers to a stochastic model where all cells within a tumor can contribute equally to the initiation and maintenance of the tumor mass. The second mechanism on the other hand, arranges cancer cells in a hierarchical model, where a subgroup of cells have similar qualities and duties to those of stem cells found in normal tissues, in being clonogenic and capable of continuously regenerating other tumor cells. These CSCs are supposed to be found in a special niche, and are behind the resistance to anti-cancer treatments, as well as being actively involved in metastasis [11]. The exact percentage of CSCs within various tumors is still undetermined, but there are many reports claiming that this percentage is under 1:1000 [12-15]. However, some more recent reports suggest that the numbers of CSCs might be significantly higher $[16,17]$.

Among the mounting body of evidence, supporting the CSC model is the long standing observation that cancer cells have a clearly variable potential to start new tumors. This is usually most clear upon injection of such cells into immunodeficient nude mice. Different cancer cell populations, grouped by their specific surface marker profiles, have wide differences in their abilities to form new tumor masses [18]. A good example here is the repeatedly successful xenograft tumor induction in mice when injecting colorectal cancer cells expressing the CD133 surface marker, a known CSC marker, as compared to CD133cells which fail to form tumors. Bao and coworkers investigated the possible role of CSCs radio-resistance in glioma patients using short term cell cultures from primary glioma xenografts and primary patient glioblastoma specimens [19]. This study showed that the population of CSCs was enriched noticeably by irradiation and that those irradiated CSCs had survival advantages relative to non-CSCs. Moreover, the irradiated CSCs showed enrichment of CD133+ cells in comparison to parent cells [20].

It is needless to say that discovering cell surface markers differentially expressed on CSC will greatly boost our understanding of the behavior of these cells by being able to better isolate, enrich, and even target them. Several such markers have indeed been described including CD44, CD133 and EpCAM. However, it is worth mentioning here that questions are being continuously raised about the specificity of and the role of these described CSC markers in various tumors. For example, Shmelkov et al. demonstrated that both $\mathrm{CD}_{133^{+}}$and
CD13- human metastatic colon cancer cells exhibit CSC properties and were able to initiate tumors thereby suggesting that CD133 could not differentiate between colon CSCs and differentiated metastatic colon cancer cells [21]. On the other hand, another study performed by Kemper and his colleagues, concluded that failure in detection of CD133 with differentiation of colon CSCs is not related to the loss in CD133 expression. Rather, post-translation modifications to the AC133 epitope of CD133 lead to changes in glycosylation and conformational changes that mask AC133 epitope detection, which is associated with CSC differentiation [22]. Such uncertainty extends to other known CSC markers, which is why scientists believe that there remains a need to discover and characterize more CSC markers that are more exclusively expressed on such cells.

\section{CSC and drug resistance}

Treatment of malignancies using conventional therapeutic regimens including chemotherapy and radiotherapy still fails to extirpate many tumors, a failure indicated usually by the relapse of tumors following initial remission. Cancer radio- or chemotherapies target mainly the highly proliferative cells and initially leads to reduction in the bulk of the tumor mass [23]. However, in many cancers, these therapies fail to prevent tumor relapse in the long-term. One explanation that is receiving wide acceptance for such recurrent relapse is the existence of CSCs, which are more resistant to the toxic effect of antitumor drugs. This explanation also reflects the inability of such conventional antitumor therapies to target CSCs [24,25] (Figure 1).

Many studies were conducted to investigate the mechanism of CSC resistance to conventional antitumor therapies. Radiation can cause damage to DNA of cancer cells resulting in induction of apoptosis pathways. However, CSCs were shown to be able to repair DNA damage more rapidly than the non-CSC populations through the activation of DNA damage checkpoints which require intact Chk1/2 kinases to arrest cancer cells and then repair the damaged DNA $[26,27]$. Woodward and coworkers also showed the role of the Wnt $/ \beta$ catenin pathway in radio-resistance of CSCs of breast cancer cell lines by enhancing the self-renewal pathway and thus increasing survival $[28,29]$. Moreover, Todaro et al. suggested a role for interleukin-4 receptor in the chemotherapeutic resistance of colon CSCs. They found that when the interleukin- 4 receptors were blocked using antibodies,
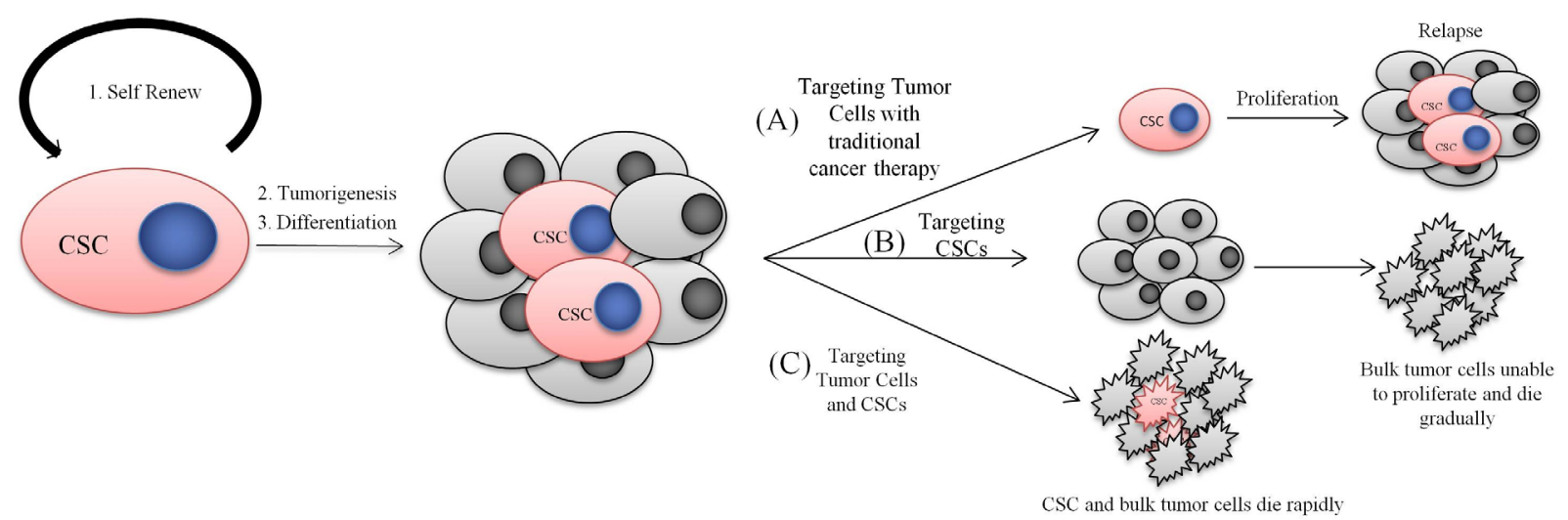

Figure 1: Schematic representation showing the effect of targeting different tumor cell populations. (A) When cancer cells are targeted by traditional cancer therapy bulk tumor cells will die while the more resistant cancer stem cells (CSCs) survive and eventually induce relapse. (B) Specifically targeting the tumor initiating CSCs will leave the bulk mass of tumor cells unable to proliferate efficiently and thus will die gradually. (C) Targeting all cancer cells including CSCs will result in rabid elimination of the tumor. Adopted from reference [20]. 
CSCs became more sensitive to chemotherapy and sustained remission was observed [30].

CSCs were also shown to have increased capacity to express drug efflux pumps such as multidrug resistance transporter1 (MDR1) and breast cancer resistance protein (BCRP) and many studies have shown the ability of such pumps to remove chemotherapeutic agents out of the cells Interestingly. Studies have implicated CSCs in angiogenesis by expression of higher levels of vascular endothelial factor (VEGF) compared to the non-CSC population. One such study was performed on CSCs from glioma models, which demonstrated an over-expression of VEGF that was associated with more vesicular and hemorrhagic tumors $[31,32]$.

\section{The emergence of aptamers}

Background: Aptamers are short single-stranded oligonucleotides of deoxyribonucleic acid (DNA) or ribonucleic acid (RNA) sequences that fold into unique secondary and tertiary structures which allow them to bind to target molecules with high affinity and selectivity.
Specific aptamers are selected from large libraries of random sequences synthesized by combinatorial chemistry. The process by which they are selected is called SELEX (Systematic Evolution of Ligands by EXponential enrichment).

The very first chapters of the aptamer story were written around 20 years ago, when two groups almost simultaneously put the building blocks of this new technology. In 1990, Szostak and Gold pioneered the in vitro evolutionary process which they termed SELEX for selection of RNA ligands that can bind the T4 DNA polymerase [33], while Ellington and Szostak developed a variety of short RNA ligands against some organic dyes. They gave these RNA ligands the name Aptamers, which they derived from the Latin word "aptus"- which means fitting and the Greak word "meros", which means particle [34].

Since its first description, there have been many modifications and variations on the SELEX procedure. In its basic format, the procedure begins with synthesis of a nucleic acid library, followed by repetitive rounds of screening and selection of sequences that can bind the

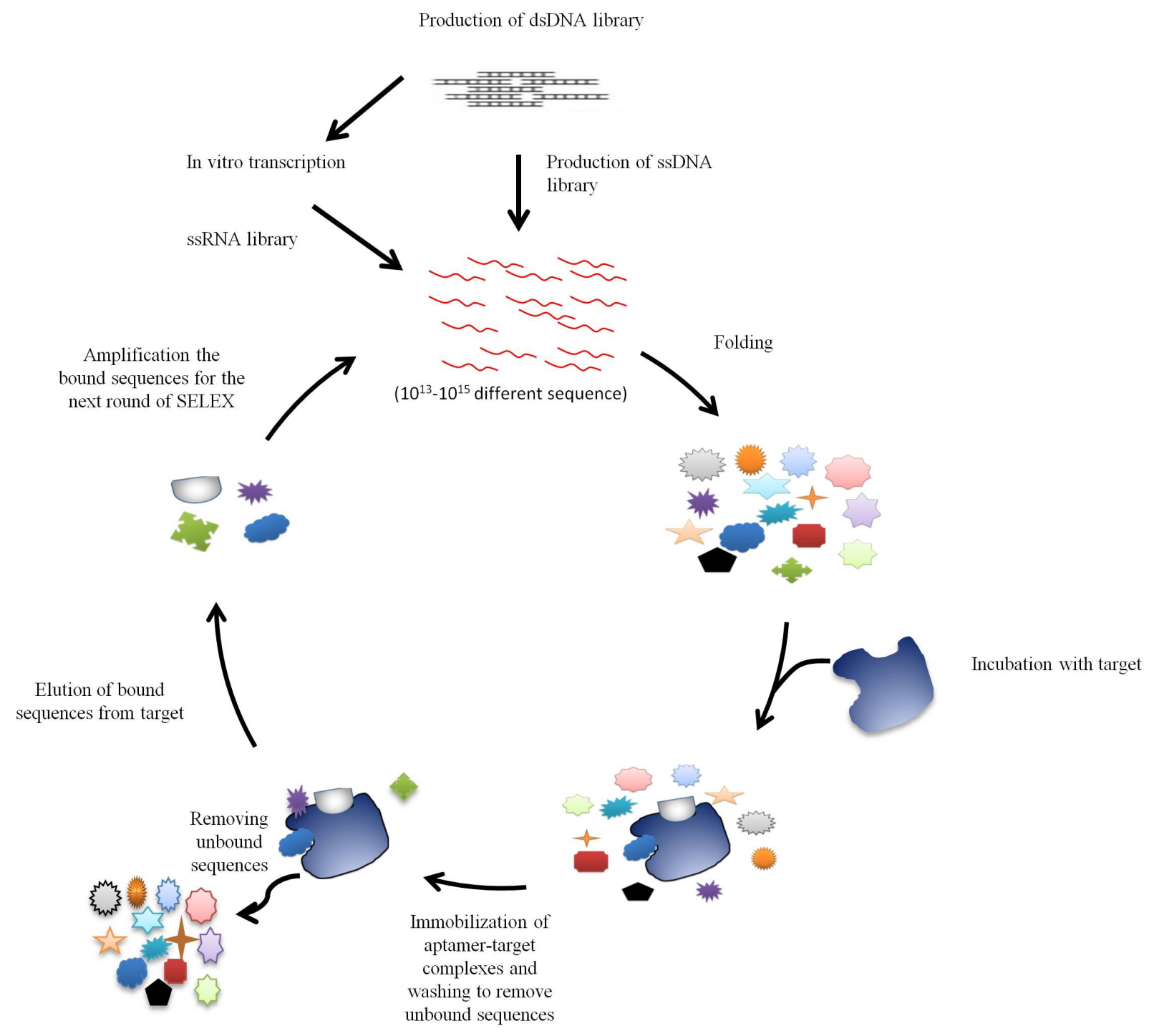

Figure 2: The SELEX process. 
desired target, after which the final group of selected aptamers is put through sequence analysis as well as structural studies of resulting aptamer-target complexes (Figure 2). The nucleic acid library could be a DNA or RNA library usually containing $10^{14}-10^{15}$ different sequences. Each sequence usually contains 20-60 nucleotide-long random region flanked by two constant regions. These fixed regions are usually designed to include a promoter region, restriction sites for cloning, and primer sites for reverse transcription and PCR amplification [35].

A classical SELEX round starts by first allowing the pool of random sequences to fold under the required experimental conditions before incubating this pool with the target molecule. The target-bound aptamers are separated from the non-binders and are then PCR amplified. This results in a new smaller library with which the next round of selections begins. These steps are repeated several times to enrich populations of aptamers that have increasing target affinity and specificity. The rounds of enrichment and amplification are continued until the pool is saturated with much fewer species of dominating aptamers. The selected candidates are then cloned and characterized. In the case of RNA aptamers, there is one main extra step which includes in vitro transcription to produce the RNA library and reverse transcription before amplifying the selected sequences [36].

In recent years, many reports described a number of new SELEX variants to meet the ever expanding applications for aptamers and improve their qualities as well as reducing time and cost of production. Examples of these variants include counter SELEX, which utilizes analogues to the target to eliminate aptamers recognizing similar targets, and thus increase the selectivity of the obtained sequences [37]. Cell specific SELEX (CS-SELEX) is another important method used to discover new cell specific surface markers. This method is particularly useful for identification of cancer-specific markers, where the aptamer library is alternated between the cancer cell and its normal counterpart to select sequences capable of exclusively recognizing the abnormal cell [38]. More recently, there have been several modifications on the classical manual SELEX procedure towards more automation and utilization of modern technology [39].

Owing to their many useful characteristics, aptamers are being used in a wide variety of applications including diagnostics, purification processes, drug discovery, and therapeutics [40]. In diagnostics, aptamers were shown to fit in a variety of platforms where they can substitute for monoclonal antibodies. Moreover, aptamer-based assays have proven to be very sensitive reaching considerably low detection limits [41]. As aptamers are chemically synthesized, they can be easily modified at their $3^{\prime}$ or $5^{\prime}$ end with many useful functional groups or conjugates. Linking aptamers to molecules like biotin would allow their immobilization on a carrier surface where they function as capture ligands [42]. Recently, several bio-analytical methods have used nucleic acid probes to detect specific sequences of RNA or DNA targets through hybridization. These specific nucleic acids have been used in many analytical applications including affinity chromatography, capillary electrophoresis, mass spectrometry, biosensors, and Enzyme Linked Oligonucleotide Assay (ELONA) [43].

The use of aptamers in drug discovery holds great potential as they are particularly suited as artificial ligands for target validation applications in the context of high-throughput screening (HTS). Such an approach is especially useful when the natural ligand is not known or the exact structure is unavailable. In addition, such screening assays are useful when new motifs on a protein are being studied, looking for small-molecule drugs with potentially novel mechanisms of action [40].

In therapeutic applications, aptamer technology received a great confidence boost in 2004 with the first FDA approval of a therapeutic aptamer called Macugen (pegaptamib) by Pfizer and Eyetech, which is

Aptamers

\begin{tabular}{|c|c|}
\hline Advantages & Disadvantages \\
\hline $\begin{array}{l}\text { - Selection performed in vitro and the conditions can be easily controlled to meet the } \\
\text { perspective applications }\end{array}$ & $\begin{array}{l}\text { Sensitivity to degradation by nucleases that require extra chemical modifications } \\
\text { to the oligonucleotide }\end{array}$ \\
\hline - Wide range of targets, starting from ions to whole living cells & $\begin{array}{l}\text { - Rapid circulation clearance that require conjugation with other molecules such as } \\
\text { PEG to decrease clearance rate. }\end{array}$ \\
\hline \multicolumn{2}{|l|}{ - High binding affinity of (pecomolar to nanomolar concentrations) } \\
\hline \multicolumn{2}{|l|}{ - High specificity } \\
\hline \multicolumn{2}{|l|}{ - Chemically synthesized and easy to reproduce } \\
\hline \multicolumn{2}{|l|}{ - Renature easily after denaturation } \\
\hline \multicolumn{2}{|l|}{ - Long shelf life } \\
\hline \multicolumn{2}{|l|}{$\begin{array}{l}\text { - Easy to conjugate with nanopartecles and active motifs with known aptamer } \\
\text { orientation }\end{array}$} \\
\hline \multicolumn{2}{|l|}{ - Can be expressed intracellularly as intramers } \\
\hline \multicolumn{2}{|l|}{ - Low toxicity and immunogenicity } \\
\hline \multicolumn{2}{|l|}{ - Relative low cost of production } \\
\hline \multicolumn{2}{|l|}{ - Small compared to antibodies } \\
\hline \multicolumn{2}{|l|}{ - Availability of antidots } \\
\hline \multicolumn{2}{|c|}{ Antibodies } \\
\hline Advantages & Disadvantages \\
\hline - High binding affinity & - Immunogenic \\
\hline - High binding specificity & - Made in vivo, thus limit the control over production \\
\hline - Production technology and applications are established in research and clinically & - Produced only against immunogenic molecules, which limits the range of targets \\
\hline \multirow[t]{4}{*}{ - Low clearance rate from the body } & - Denaturation is irreversible. \\
\hline & - Short half life. \\
\hline & - Sensitive to conditions such as temperature and $\mathrm{pH}$ \\
\hline & - High cost of production \\
\hline
\end{tabular}

Table 1: Summary comparison of the advantages and the disadvantages of aptamers and antibodies. 
an anti-VEGF aptamer used to treat age-related macular degeneration of the eye. For their clinical use, scientists had to modify aptamers in many ways to overcome a number of obstacles. One of these obstacles is that aptamers, especially RNA aptamers, are susceptible to nuclease degradation. However, the stability of RNA aptamers in vivo can be easily enhanced by simple chemical modifications including 2'-fluoro pyrimidine, 2'-O-methyl nucleotides and 3 ' end cap modifications [44]. Other modifications on aptamers have been used to slow down the rapid renal clearance of such small $(5-25 \mathrm{kDa})$ and highly water soluble molecules. Such enhancement of bioavailability has been achieved by linkage with cholesterol or polyethylene glycol as anchor groups [45]. Aptamers developed as therapeutics have a wide range of actions including anti-infectives, anti-coagulants, anti-inflammatory, anti-angiogenesis, anti-proliferative, and immune modulators [46,47].

Aptamers versus monoclonal antibodies: Although different in chemistry, structure, production method and other aspects, the nearest class of molecules that have comparable functional properties and similar diagnostic and therapeutic applications would be monoclonal antibodies. Aptamers have been shown to bind their targets with high affinities with Kds in the picomolar to nanomolar range, and with very high specificity enabling them to distinguish between very closely related targets. For example, anti-L-selectin aptamers are 8,000-15,000 fold and 200-500 fold more specific to their target as opposed to P-selectin and E-selectin, respectively [48]. Moreover, researchers involved in aptamer technology always like to emphasize some advantages that aptamers have over monoclonal antibodies. For example, unlike antibodies which are usually produced by the complex and labor intensive Hybridoma technique which involves extensive tissue culture work and the immunization of a mammalian host, aptamers are produced by SELEX which is a relatively simple in vitro chemical method. In SELEX, conditions like $\mathrm{pH}$, ionic strength, temperature and the process of selection can be easily controlled to achieve efficient selection. This also means that aptamers can be developed not just against immunogenic proteins, but also against a wide range of target molecules including drugs and other small molecules, and even against whole cells [41].

One clear advantage, aptamers have as therapeutic agents, is that they are largely non-immunogenic as studies on humans and mammalians showed no antigenic response. In addition, aptamers have also been shown to exhibit no or little toxicity. The best data in this regard comes from work on the FDA approved therapeutic aptamer Macugen, which has been used safely for years [49-51]. Nevertheless, a recent study showed that ssDNA aptamers might provoke an immune response after systemic administration into fresh human blood. Therefore, preclinical experiments to investigate such immune responses against each therapeutic aptamer are highly recommended [52]. However, and even in the unlikely event of aptamer induced adverse pharmacological reactions, such reactions can be easily controlled by an antidote which can simply be another single stranded oligonucleotide that has the antisense base sequence. The Watson-Crick base pairing between the aptamer and the antidote would alter the shape of aptamer and thus inhibit the binding of the aptamer to its target [45] (Table 1).

Aptamers for targeted drug delivery: Several nucleic acid aptamers were evolved to target cell specific biomarkers including those on the surface of tumor cells. Such aptamers have been used for targeted delivery of diverse molecules including chemotherapeutic agents, nanoparticles, drug-encapsulated liposomes, small interfering RNA (siRNA), toxins, and radioactive materials [53-58]. The mechanism of delivery is based on the specific interaction between the aptamer and its cellular membrane receptor or antigen, which might then enhance the internalization and accumulation of therapeutic agents into the targeted cells. Such controlled delivery will thereby result in increasing drug potency and efficacy as well as decrease unwanted toxic side effects.

One representative example was reported by Lupold et al. who isolated modified $2^{\prime}$-fluoro-RNA aptamers against the prostate specific membrane antigen (PSMA) which is highly expressed on human prostate cancer cells [59]. Using these aptamers, many other studies have successfully attempted to conjugate these aptamers with various active motifs and nanocarrier systems to mediate cell specific targeted delivery for therapeutic and imaging purposes of PSMA positive cells. These conjugates include nanoparticles, small interfering RNA (siRNA) and antitumor drugs. As for nanoparticles, Farokhzad et al. developed Docetaxel encapsulated nanoparticles functionalized with a specific aptamer (A10) to target PSMA expressing cells (denominated DtxlNP-Apt) [60]. The nanoparticle was formulated with Poly (lactic-coglycolicacid) (PLGA) co-polymered with Poly ethylene glycol (PEG). PLGA is considered as one of the most successful biodegradable and biocompatible polymer used to formulate nanoparticles and is approved by the US Food and Drug Administration (FDA) and the European Medical Agency (EMA) for many drug delivery systems in humans [61]. Moreover, the addition of polyethylene glycol on the surface of nanoparticles was used to improve the pharmacokinetics of the involved therapeutics by increasing the circulation half-life of these nanoparticles [62]. The results of this study demonstrated the ability of the Dtxl-NP-Apt bioconjugate to enhance in vitro cellular cytotoxicity to LNCaP PSMA expressing cells and increase tumor reduction in LNCaP xenograft nude mice, as compared to the therapeutic potency of Dtxl-NP or Dtxl alone [60].

siRNA is considered a promising and attractive therapeutic molecule that is able to specifically knockdown and silence gene expression via RNA interference. siRNA advantages include specificity of targeting, low immunogenicity, and simplicity of design [63]. However, cells are unable to efficiently take up siRNA and it requires a delivery vehicle for it to reach its intracellular targets [64]. To overcome this problem, aptamers have been successfully used to increase the internalization of siRNA by a receptor-mediated mechanism. McNammara et al. developed an aptamer-siRNA chimeric RNA molecule by covalent conjugation of the anti PSMA aptamer (A10) with siRNA molecules that target the two tumor survival genes, Polo-Like Kinase 1 (Plk1) and BCL2 [65]. The results of this study showed the ability of the A10siRNA chimera to bind specifically to LNCaP cells (PSMA positive) while failing to bind PC-3 cells (PSMA negative). Moreover, silencing of targeted genes (Plk1 and BCL2) was shown to be specific and comparable with the binding/uptake of A10-siRNA chimera to the level of PSMA expression on LNCaP cells. The efficacy of the A10-siRNA chimeras in tumor reduction was tested by intratumoral injection these chimeras into xenografted tumor models of prostate cancer. A marked regression in the tumor volume was observed in PSMA-positive tumors while treating PSMA-negative control tumors with the same A10-siRNA chimeras showed an increase in tumor volume thereby indicating the specificity and therapeutic potency of the A10-siRNA chimeras in invivo models [65]. In another study, a second generation of the A10siRNA chimera (A10-Plk1) was developed to be more cost effective and easier to synthesize. The 79 nucleotides full length A10 aptamer was truncated into a 39 nucleotides length aptamer (A10-3.2 aptamer) without significant change in the binding affinity and specificity to PSMA on target cells. This and other modifications performed on the aptamer-siRNA chimera enhanced the silencing activity and specificity of siRNA inside the cells. Moreover, 20 KD PEG was conjugated to 5' terminal of RNA chimera to increase the circulating half-life. The end 
results of this study showed that the second generation of A10-3.2siRNA-PEG chimera was more optimal for systemic administration rather than intratumoral injections and has a greater gene silencing capability in vivo [66].

Combinations of two or more therapies that hold additive or synergistic effect are common in cancer treatment to overcome the development of drug resistant and decrease therapeutic doses thereby decreasing undesirable side effects [67]. Kim et al. investigated the synergistic effect of short hairpin RNA against the $\mathrm{Bcl}-\mathrm{Xl}$ anti apoptotic protein and Doxorubicin, the anti-cancer chemotherapy drug, using the anti-PSMA aptamer (A10) conjugated to polyplexes composed of polyethyleneimine (PEI) grafted to polyethylene glycol (PEG) to form the nanoplatform shRNA/PEI-PEG-APT/DOX. The anti-cancer effect of this nanoplatform was evaluated on LNCaP cell line and PC-3 cell line and the cellular viability results showed high specific inhibition of shRNA/PEI-PEG-APT/DOX to PSMA expressing cells compared to the mixture of Doxorubicin and shRNA alone indicated by a 17 -fold decrease in the IC50 value of shRNA/PEI-PEG-APT/DOX compared to the IC50 values for the Doxorubicin and shRNA mixture [68].

Another interesting example is the development of the DNA aptamer (AS1411) that recognizes and binds with high affinity and specificity to nucleolin (NCL). This AS1411 aptamer was shown to be able to cross the cell membrane via NCL-mediated internalization [69]. NCL is a multifunctional protein involved in many cellular pathways including DNA metabolism and RNA regulatory mechanisms. NCL has been linked to many pathological abnormalities including cancer and viral infection [70]. Therefore, the AS1411 aptamer could be useful in targeting cancer cells that over-express NCL. Indeed, in 2009, Cao et al. developed an AS1411aptamer functionalized liposome with Cisplatin encapsulated inside for cancer-cell-specific targeting. Cisplatin is one of the most potent anticancer chemotherapies that work by inducing DNA damage. However, Cisplatin lacks specificity and can cause severe and widespread side effects on normal cells [71]. The cytotoxic effect of the aptamer-liposome-cisplatin complexes were evaluated using MCF7 (NCL positive) and LNCaP (NCL negative) cell lines and the results showed a greater damage to MCF-7 cells compared to LNCaP cells. The cytotoxic effect of cisplatin on MCF-7 and LNCaP was reported to have $\mathrm{IC}_{50}$ values of $28 \mu \mathrm{M}$ and $5.95 \mu \mathrm{M}$, respectively. The AS1411 anti-NCL aptamer was first tagged with a cholesterol moiety and then inserted into the hydrophobic lipid part of the liposome. Interestingly, in the same study, the authors described a complimentary DNA strand to AS1411 aptamer that works as an antidote and can reverse the action of aptamer-mediated targeted drug delivery [72].

In addition, several other cell-specific aptamers that were developed against tumor markers such as MUC-1, Protein Tyrosine Kinase 7 (PTK7) and Epithelial Growth Factor Receptor (EGFR) among others clearly demonstrated the great potential for aptamer mediated delivery systems [8].

Aptamers for molecular recognition and biomarker discovery: Since the early development of the SELEX method, studies have been conducted to utilize aptamers in diagnostic applications owing to their promising properties as highly versatile molecular recognition agents. As mentioned earlier, and similar to monoclonal antibodies, aptamers can bind to their targets with high affinity and specificity enabling them to detect very low target concentrations and to discriminate between very closely related targets. For example, Jenison et al. selected an anti-theophylline aptamer that was 10,000 fold more likely to bind theophylline as opposed to caffeine, two structurally similar molecules which differ by only a single methyl group [37]. In another example,
Geiger et al. described a binding affinity of an anti-L-argenin RNA aptamer that was 12,000 times stronger than that for its binding to D-argenin [73]. Moreover, Jeong et al. identified an RNA aptamer that binds around 50 fold better to mutant KRAS having a point mutation in codon 12 (KRASV12), compared to the wild type KRAS protein [74].

The SELEX methodology can be efficiently utilized for the selection of aptamers against a wide variety of targets making it a powerful tool for different biomedical applications. Selection of aptamers against characterized purified protein targets can be easily performed by simple conventional SELEX. However, the main limitation with such SELEX against pure targets is that the selected aptamers frequently fail to bind the protein target in its native cellular state [5]. One modification was suggested to solve this issue by developing a complex target selection method to select aptamers against proteins in a complex mixture of targets prepared from membrane preparations or whole living cells, thus enabling the aptamers to bind its target in their preferred native state. Moreover, one important advantage to this method is the ability to select aptamers against unknown targets without detailed previous knowledge and characterization of the target, which makes it possible to discover and identify cell specific markers on diseased cells such as cancer cells and viral infected cells $[5,7]$.

Cell-SELEX is a variant procedure used to select aptamers against targets on living cells including parasites, bacteria, viruses, stem cells, and tumor cells. Shangguan et al. developed a cell-based aptamer selection strategy to select ssDNA aptamers that bind molecular signatures on tumor cell lines. Two selected hematopoietic cancer cell lines were used as a model, the $\mathrm{T}$ cell acute lymphoblastic leukemia (ALL) cell line CCRF-CEM was the target cell line and the Burkitt's lymphoma Ramos cell line, was used for counter selection to remove aptamers that bind to shared molecules on the surface of those closely related cell lines [75]. Following successful selection of specific aptamers, further investigation is usually performed using proteomics to characterize the marker that is uniquely expressed on the targeted cell line. One good example on the potency of this procedure is the tyrosine protein kinase-like 7 (PTK7) protein which was discovered and identified as a signature biomarker for $\mathrm{T}$ cell acute lymphoblastic leukemia making it a good candidate for specific targeting of these cells. Moreover, and in similar experiments using cell-SELEX, many other biomarkers were discovered including the glycoprotein Tenascin-C, which was discovered on the glioblastoma-derived cell line (U251), and the immunoglobulin $\mu$ heavy chain (IGHM) as a biomarker for Burkitt's lymphoma using Ramose cell lines [76,77].

\section{Aptamers against specific CSC markers}

Selection of aptamers against specific markers of CSCs provides a powerful tool for a myriad of applications that would help to better understand the biological behaviour of these cells. Such specific aptamers can be utilized in various assay platforms to isolate, enrich, and identify CSCs for analytical and diagnostic purposes in addition to the potential of being used in targeted drug delivery. The most crucial step towards the successful development of such applications is the characterization of specific markers for CSCs. Indeed, in recent years, and with the identification of such markers, reports started to emerge describing the selection of CSC specific aptamers. Following is a brief description of the available related literature.

Aptamers against epithelial cell adhesion molecule (EpCAM): The epithelial cell adhesion molecule (EpCAM; CD326) is a transmembrane glycoprotein originally discovered on colon carcinomas [78]. EpCAM has been described as a CSC marker of mammary, 
colorectal and pancreatic cancers [79]. EpCAM is also known to be a tumor associated antigen because of its frequent discovery in a wide variety of carcinomas [80]. Currently, the overexpression of EpCAM on most carcinoma types is used as a diagnostic marker with some prognostic value and as a target in antibody based clinical trials [81]. EpCAM is widely distributed in normal human epithelial tissues but is typically overexpressed in a variety of carcinomas including most human adenocarcinomas as well as squamous cell carcinomas. In the case of breast and ovarian cancers, EpCAM mRNA was found to be more than 100-fold overexpressed relative to normal epithelial tissues. Its overexpression usually correlates with a decrease in survival in breast and ovarian cancers $[82,83]$.

Several clinical trials were performed to target EpCAM expressing tumor cells with monoclonal antibodies. Such trials showed inconsistent results, which was related in part to the large size, affinity, and high immunogenicity of antibodies [80,84]. Therefore, selection of small aptamers that can target EpCAM could be a good alternative for these antibodies. Shigdar et al. isolated modified RNA aptamers that can bind to EpCAM from a random oligonucleotide library. The highest affinity selected EpCAM aptamer did bind to EpCAM positive gastric carcinoma cells Kato III with a Kd of $211 \pm 36.2 \mathrm{nM}$. The full length EpCAM aptamer (73 nt long) was further truncated into smaller oligos by performing two rounds of truncation. The first truncation ended with the EpDT1 aptamer (43 nt long) and the second round ended with the EpDT3 aptamer (19 nt long), the Kds of truncated aptamers were $85.7 \pm 24$ and $12.0 \pm 6.5 \mathrm{nM}$ (using Kato II cells), respectively. Interestingly, the apparent $\mathrm{Kd}$ increased from 12 $\mathrm{nM}$ to $54.5 \mathrm{nM}$ when EpDT3 was labeled with the DY647 fluorophore. This EpDT3- DY647 labeled aptamer was incubated with a number of human cancer cells derived from breast, colorectal, and gastric cancers that express EpCAM, and the fluorescent intensity from each cell line was analyzed using flow cytometry. The results corresponded well with the known EpCAM expression levels for each of the representative cell lines. Moreover, confocal microscopic analysis showed EpDT3DY647 to be internalized after binding through receptor mediated endocytosis [85]. This study describes the first selection of modified RNA aptamers that can specifically bind the EpCAM CSC marker and successfully induce internalization by endocytosis. This should prompt further investigation of the potency of the selected aptamer using in vivo models, and probably the conjugation of this aptamer to drug nanocarriers or imaging systems for various clinical purposes.

Aptamers against the HA domain of CD44: CD44 comprises a family of glycoproteins encoded by a single gene [86], which vary in size due to $\mathrm{N}$ - and $\mathrm{O}$-glycosylation and insertion of alternatively spliced variable exon products in the extracellular domains of the molecule [79]. CD44 is a multi-structural and multi-functional cell surface molecule involved in cell proliferation, cell differentiation, cell migration, angiogenesis and presentation of cytokines, chemokines and growth factors to the corresponding receptors. It is a unique adhesion molecule and several studies have revealed that CD44 is over expressed at the mRNA and protein levels in most types of solid tumors [87].

$\mathrm{CD} 44$ is the main receptor for hyaluronic acid (HA). Therefore, HA was considered as a promising carrier for active targeting of therapeutic and imaging molecules into CD44 expressing tumor cells. However, HA lacks specificity as it can bind to several proteins other than the CD44 receptor [88]. Based on that, selection of aptamers that bind specifically to the extracellular part of CD44 could be a promising agent for active targeting. DNA thioaptamers (TAs) were selected to bind the hyaluronic acid binding domain (HABD) of the CD44 protein with high specificity and affinity. The binding affinities of the selected TAs were in nanomolar concentrations (180-295 nM), which is higher than the affinity of the natural ligand, hyaluronic acid (micromolar concentrations). The selected TAs did bind successfully to CD44 positive human ovarian cancer cell lines (SKOV3, IGROV, and A2780) but failed to bind the CD44 negative NIH3T3 fibroblast cell line [89].

Our group has recently managed to successfully select high affinity RNA aptamers against standard CD44 (CD44s) [90]. We used a 2'-F-pyrimidine modified RNA library to isolate RNA aptamers against GST tagged human recombinant full-length CD44 protein. The binding specificities for one of the selected RNA aptamers (Apt1) was assessed using representative breast cancer cell lines expressing CD44, namely MDA-MB-231, MCF7 and T47D. The selected RNA aptamer was found to interact specifically with such cancer cells when analysed by flow cytometry and fluorescent microscopy with different intensities of fluorescence, reflecting the level of CD44 expression on the surface of these cells. We also tested the binding affinity for Apt1 with the CD44 protein and our results have clearly shown a high binding affinity with $\mathrm{Kd}$ in the nanomolar range. This work further demonstrates that the CD44 receptor can be efficiently targeted by aptamers with affinities and specificities that are comparable or even higher than that of hyaluronic acid, the natural CD44 ligand. Therefore, it is expected that such aptamers can be successfully implicated in diagnostic and therapeutic applications.

RNA aptamers targeting CD133: CD133 is a cell surface glycoprotein with five transmembrane domains and is considered as a universal marker of normal hematopoietic and organ-specific stem cells [91]. It has gained more prominence as a marker of CSCs in solid primary tumors such as medulloblastomas and glioblastomas and subsequently of CSCs in a growing number of cancers of epithelial tissues. It has also been identified in CSCs of many other tissues, including prostate cancer and colon cancer $[92,93]$.

Shigdar et al. identified two 2 '-fluoropyrimidine modified RNA aptamers, CD133-A15 and CD133-B19. The CD133-A15 aptamer showed the ability to bind the AC133 epitope of CD133 while the CD133-B19 aptamer was able to bind to the extracellular domain of CD133. These selected aptamers also managed to successfully bind CD133 positive cell lines (HT-29 and Hep3B) with nanomolar affinities, while failing to bind negative CD133 cells lines (HEK293 and T98G) [94]. This study demonstrates the ability of selected aptamers to bind to certain epitopes of the tumour marker that appears on more primitive CSCs thereby providing more specificity in targeting. Still, an in vivo study that investigates the potency of such selected aptamer could provide further valuable information for this promising work and would stimulate similar efforts to select and use other aptamers against other targets.

Aptamers identified against brain CSCs: A recent study performed by Kim et al. used the cell-SELEX technique to isolate DNA aptamers against the glioblastoma brain tumour initiating cells with high affinity and specificity. This study used glioblastoma tumor initiating cells for positive selection, whereas the negative cells used for counter selection were non-tumour initiating cells and human neural progenitor cells (NCPs). Microarray analysis for the positive aptamerbound tumour initiating cells showed gene expression profiles and molecular pathways that promote tumorgenesis [95]. Glioblastoma is classified as one of the most lethal cancers. Therefore, the selection of aptamers that can specifically bind the CSCs of Glioblastoma using cell-SELEX could help discover and characterize new biomarkers for 
these cells which might eventually be used for effective targeted drug delivery against such tumor cells.

Aptamers against prostate cancer stem cells: Prostate cancer is considered one of the leading causes of cancer death worldwide. Sefah et al. applied cell-SELEX to select DNA aptamers that successfully targeted prostate CSCs. This study used the DU145 prostate cancer cell line for positive selection, which ended up with two main panels of aptamers. The first panel ( 3 aptamers) bound to $90 \%$ of cells while the second panel (5 aptamers) bound to $15 \%$ of cells. Further characterization for the $15 \%$ of cells that did bind to the second panel showed characteristics of prostate CSCs including high expression levels of E-cadherin and CD44, high aldehyde dehydrogenase 1 activity, spheroid growth in non adherent conditions, and the ability of this portion of cancer cells to initiate tumours in immune-compromised mice. These results demonstrate the potential of using the first panel for diagnostic purposes and the second panel for targeting prostate CSCs [96]. In further work performed by Wang et al. two of the selected aptamers were conjugated to gold nanorods (AuNRs) for targeted thermotherapy of prostate cancers. One of those aptamers did bind to all DU145 while the second one did bind to the CSC portion of DU145 cells. The results of this study open the doors towards high specificity targeting and efficient inhibition and control of prostate CSCs [97].

Targeting the epidermal growth factor receptor (EGFR): The Epidermal Growth Factor Receptor (EGFR) is a member of a receptor tyrosine kinase family of four kinases including EGFR, ErbB2, ErbB3, and ErbB4. Normally, this family play a role in regulation of cell proliferation, apoptosis, and angiogenesis. Gene over expression or activating mutations in EGFR have been linked to many cancers where they played key roles in tumoregenesis, cell proliferation, matastasis, and drug resistance [98]. Recently, over-expression or activating mutations of EGFR were found to play a role in the maintenance and survival of CSCs by inducing the expression of CSC markers in head and neck squamous cell carcinomas [99]. Interestingly, recent evidence showed the co-localization of CD44 with EGFR on head and neck squamous cell carcinomas (HNSCC). Inhibition of CD44 using siRNA resulted in down regulation by phosphorylation of EGFR thereby indicating a crosstalk between CD44 and EGFR signalling pathways [100]. Aptamers have been selected against EGFR and showed a promising potency in inhibiting this receptor. An interesting study performed by Esposito et al. described the selection of nuclease resistant RNA aptamer (named CL4) that is able to bind specifically EGFR cancer expressing cells with an affinity of $10 \mathrm{nM}$. This study showed that CL4 is able to induce apoptosis in EGFR positive cells while having no effect on EGFR negative cells. Moreover, CL4 aptamer inhibition efficacy was compared with two anticancer agents, gefitinib and cetuximab, using the A549 lung cancer cell line and showed sensitivity to the CL4 aptamer at $200 \mathrm{nM}$ concentrations while being resistant to gefitinib and cetuximab at $0.1-10$ and $0.05-1 \mu \mathrm{M}$ concentrations, respectively. In addition, the CL4 aptamer and cetuximab showed synergy when administered into a xenograft model of non-small lung carcinoma compared to each drug alone [101]. This study demonstrates the possibility to evolve aptamers that can overcome cancer cell resistance, especially CSCs, to conventional therapeutics.

\section{Future perspectives}

The CSC hypothesis has been formulated based on experiments demonstrating the ability of a subpopulation of cancer cells to initiate and maintain tumors. These cells were shown to resist conventional anti-cancer therapies and induce relapse. The development of strategies to isolate and characterize CSCs is essential to better understand their behavior, and this is largely dependent on the identification of more specific marker proteins expressed on these cells. Although some markers have been shown to be highly expressed on CSCs, none of them has the required optimal exclusiveness of expression, which remains as one of the major challenges facing researchers. The cell-SELEX method comprises a powerful method that can accelerate the discovery of such new specific CSCs biomarkers, which should facilitate the development of effective specific targeting ligands and platforms for diagnostic and therapeutic purposes.

Although monoclonal antibodies are currently the clear first choice candidate to be incorporated into the new generation of the "smart" targeted anticancer drugs, aptamers are expected to be their serious future contender and the coming few years may witness some fierce competition between the two classes of molecules. Indeed, year after year, more clinical trials involving aptamers are underway, and this growing trend is supported by the increasing number of new biotech companies investing in this new technology.

The field of aptamers is maturing and is coming of age and one good indicator in this regard is the exponential increase in the annual number of related publications witnessed over the last decade. The technology is evolving rapidly and is becoming more accessible by the introduction of new developments including for example the automation of the SELEX method making the selection process faster and more reproducible, plus the various modifications on the different components of the nucleic acid basic structure to enhance the properties of aptamers as therapeutic molecules. With such rapid evolution of the aptamer technology and the expansion of its use in applications related to CSCs, we could witness some promising breakthroughs in this field in the near future.

\section{Acknowledgment}

We wish to thank the Hamdi Mango Center for Scientific Research (HMCSR) and the Deanship of Scientific Research (DSR) at the University of Jordan, for their continued support of our research on aptamers.

\section{References}

1. World cancer report (2008) World Health Organization (WHO).

2. Vermeulen L, de Sousa e Melo F, Richel DJ, Medema JP (2012) The developing cancer stem-cell model: clinical challenges and opportunities. Lancet Oncol 13 e83-89.

3. Vermeulen L, Sprick MR, Kemper K, Stassi G, Medema JP (2008) Cancer stem cells--old concepts, new insights. Cell Death Differ 15: 947-958.

4. Clevers $H$ (2011) The cancer stem cell: premises, promises and challenges Nat Med 17: 313-319.

5. Ohuchi S (2012) Cell-SELEX Technology. Biores Open Access 1: 265-272.

6. Keefe AD, Pai S, Ellington A (2010) Aptamers as therapeutics. Nat Rev Drug Discov 9: 537-550

7. Shamah SM, Healy JM, Cload ST (2008) Complex target SELEX. Acc Chem Res 41: 130-138.

8. Zhang $\mathrm{Y}$, Hong H, Cai W (2011) Tumor-targeted drug delivery with aptamers Curr Med Chem 18: 4185-4194

9. Magee JA, Piskounova E, Morrison SJ (2012) Cancer stem cells: impact heterogeneity, and uncertainty. Cancer Cell 21: 283-296.

10. Visvader JE (2011) Cells of origin in cancer. Nature 469: 314-322.

11. Schwarz-Cruz-y-Celis A, Meléndez-Zajgla J (2011) Cancer stem cells. Rev Invest Clin 63: 179-186.

12. Ricci-Vitiani L, Lombardi DG, Pilozzi E, Biffoni M, Todaro M, et al. (2007) Identification and expansion of human colon-cancer-initiating cells. Nature 445: $111-115$.

13. Al Hajj M, Wicha MS, Benito-Hernandez A, Morrison SJ, Clarke MF (2003) 
Citation: Ismail SI, Alshaer W, Ababneh N, Fattal E (2013) Aptamers: Promising Molecules for Cancer Stem Cell Targeting. J Mol Genet Med 7: 90 doi: 10.4172/1747-0862.1000090

Prospective identification of tumorigenic breast cancer cells. Proc Natl Acad Sci USA 100: 3983-3988

14. Eramo A, Lotti F, Sette G, Pilozzi E, Biffoni M, et al. (2008) Identification and expansion of the tumorigenic lung cancer stem cell population. Cell Death Differ 15: $504-514$

15. O'Brien CA, Pollett A, Gallinger S, Dick JE (2007) A human colon cancer cell capable of initiating tumour growth in immunodeficient mice. Nature 445: 106-110.

16. Kelly PN, Dakic A, Adams JM, Nutt SL, Strasser A (2007) Tumor growth need not be driven by rare cancer stem cells. Science $317: 337$.

17. Quintana E, Shackleton M, Sabel MS, Fullen DR, Johnson TM, et al. (2008) Efficient tumour formation by single human melanoma cells. Nature 456: 593-598.

18. Dalerba P, Cho RW, Clarke MF (2007) Cancer stem cells: models and concepts. Annu Rev Med 58: 267-284.

19. Bao S, Wu Q, McLendon RE, Hao Y, Shi Q, et al. (2006) Glioma stem cells promote radioresistance by preferential activation of the DNA damage response. Nature 444: 756-760.

20. Bao S, Wu Q, Sathornsumetee S, Hao Y, Li Z, et al. (2006) Stem cell-like glioma cells promote tumor angiogenesis through vascular endothelial growth factor. Cancer Res 66: 7843-7848.

21. Shmelkov SV, Butler JM, Hooper AT, Hormigo A, Kushner J, et al. (2008) CD133 expression is not restricted to stem cells, and both CD133+ and CD133metastatic colon cancer cells initiate tumors. J Clin Invest 118: 2111-2120.

22. Kemper K, Sprick MR, de Bree M, Scopelliti A, Vermeulen L, et al. (2010) The AC133 epitope, but not the CD133 protein, is lost upon cancer stem cell differentiation. Cancer Res 70: 719-729.

23. Soltanian S, Matin MM (2011) Cancer stem cells and cancer therapy. Tumour Biol 32: 425-440.

24. Koch U, Krause M, Baumann M (2010) Cancer stem cells at the crossroads of current cancer therapy failures--radiation oncology perspective. Semin Cancer Biol 20: 116-124

25. Han L, Shi S, Gong T, Zhang Z, Sun X (2013) Cancer stem cells: therapeutic implications and perspectives in cancer therapy. Acta Pharm Sin B 3: 65-75.

26. Gorgoulis VG, Vassiliou LV, Karakaidos P, Zacharatos P, Kotsinas A, et al (2005) Activation of the DNA damage checkpoint and genomic instability in human precancerous lesions. Nature 434: 907-913.

27. Bartkova J, Horejsí Z, Koed K, Krämer A, Tort F, et al. (2005) DNA damage response as a candidate anti-cancer barrier in early human tumorigenesis. Nature 434: 864-870.

28. Woodward WA, Chen MS, Behbod F, Alfaro MP, Buchholz TA, et al. (2007) WNT/beta-catenin mediates radiation resistance of mouse mammary progenitor cells. Proc Natl Acad Sci U S A 104: 618-623.

29. Reya T, Clevers H (2005) Wnt signalling in stem cells and cancer. Nature 434 843-850.

30. Todaro M, Alea MP, Di Stefano AB, Cammareri P, Vermeulen L, et al. (2007) Colon cancer stem cells dictate tumor growth and resist cell death by production of interleukin-4. Cell Stem Cell 1: 389-402.

31. Wulf GG, Wang RY, Kuehnle I, Weidner D, Marini F, et al. (2001) A leukemic stem cell with intrinsic drug efflux capacity in acute myeloid leukemia. Blood 98: 1166-1173.

32. Oka N, Soeda A, Inagaki A, Onodera M, Maruyama H, et al. (2007) VEGF promotes tumorigenesis and angiogenesis of human glioblastoma stem cells. Biochem Biophys Res Commun 360: 553-559.

33. Tuerk C, Gold L (1990) Systematic evolution of ligands by exponential enrichment: RNA ligands to bacteriophage T4 DNA polymerase. Science 249 : 505-510.

34. Ellington AD, Szostak JW (1990) In vitro selection of RNA molecules that bind specific ligands. Nature 346: 818-822.

35. Dua P, Kim S, Lee DK (2008) Patents on SELEX and therapeutic aptamers. Recent Pat DNA Gene Seq 2: 172-186.

36. Beckert B, Masquida B (2011) Synthesis of RNA by in vitro transcription. Methods Mol Biol 703: 29-41.
37. Jenison RD, Gill SC, Pardi A, Polisky B (1994) High-resolution molecular discrimination by RNA. Science 263: 1425-1429.

38. Shangguan D, Cao Z, Meng L, Mallikaratchy P, Sefah K, et al. (2008) Cellspecific aptamer probes for membrane protein elucidation in cancer cells. J Proteome Res 7: 2133-2139.

39. Aquino-Jarquin G, Toscano-Garibay JD (2011) RNA aptamer evolution: two decades of SELEction. Int J Mol Sci 12: 9155-9171.

40. Proske D, Blank M, Buhmann R, Resch A (2005) Aptamers--basic research drug development, and clinical applications. Appl Microbiol Biotechnol 69: $367-$ 374

41. Jayasena SD (1999) Aptamers: an emerging class of molecules that rival antibodies in diagnostics. Clin Chem 45: 1628-1650.

42. Romig TS, Bell C, Drolet DW (1999) Aptamer affinity chromatography: combinatorial chemistry applied to protein purification. J Chromatogr B Biomed Sci Appl 731: 275-284

43. Iliuk AB, Hu L, Tao WA (2011) Aptamer in bioanalytical applications. Anal Chem 83: $4440-4452$.

44. Burmeister PE, Lewis SD, Silva RF, Preiss JR, Horwitz LR, et al. (2005) Direct in vitro selection of a 2'-O-methyl aptamer to VEGF. Chem Biol 12: 25-33.

45. Rusconi CP, Roberts JD, Pitoc GA, Nimjee SM, White RR, et al. (2004) Antidote-mediated control of an anticoagulant aptamer in vivo. Nat Biotechnol 22: $1423-1428$

46. Nimjee SM, Rusconi CP, Harrington RA, Sullenger BA (2005) The potential of aptamers as anticoagulants. Trends Cardiovasc Med 15: 41-45.

47. Nimjee SM, Rusconi CP, Sullenger BA (2005) Aptamers: an emerging class of therapeutics. Annu Rev Med 56: 555-583.

48. Jenison RD, Jennings SD, Walker DW, Bargatze RF, Parma D (1998) Oligonucleotide inhibitors of P-selectin-dependent neutrophil-platelet adhesion. Antisense Nucleic Acid Drug Dev 8: 265-279.

49. Foy JW, Rittenhouse K, Modi M, Patel M (2007) Local tolerance and systemic safety of pegaptanib sodium in the dog and rabbit. J Ocul Pharmacol Ther 23 $452-466$.

50. Drolet DW, Nelson J, Tucker CE, Zack PM, Nixon K, et al. (2000) Pharmacokinetics and safety of an anti-vascular endothelial growth facto aptamer (NX1838) following injection into the vitreous humor of rhesus monkeys. Pharm Res 17: 1503-1510.

51. Gryziewicz L (2005) Regulatory aspects of drug approval for macular degeneration. Adv Drug Deliv Rev 57: 2092-2098.

52. Avci-Adali M, Steinle H, Michel T, Schlensak C, Wendel HP (2013) Potentia capacity of aptamers to trigger immune activation in human blood. PLoS One 8: e68810.

53. Chu TC, Twu KY, Ellington AD, Levy M (2006) Aptamer mediated siRNA delivery. Nucleic Acids Res 34: e73

54. Kang H, O'Donoghue MB, Liu H, Tan W (2010) A liposome-based nanostructure for aptamer directed delivery. Chem Commun (Camb) 46: 249-251.

55. Farokhzad OC, Karp JM, Langer R (2006) Nanoparticle-aptamer bioconjugates for cancer targeting. Expert Opin Drug Deliv 3: 311-324.

56. Chu TC, Marks JW 3rd, Lavery LA, Faulkner S, Rosenblum MG, et al. (2006) Aptamer:toxin conjugates that specifically target prostate tumor cells. Cance Res 66: 5989-5992.

57. Bagalkot V, Farokhzad OC, Langer R, Jon S (2006) An aptamer-doxorubicin physical conjugate as a novel targeted drug-delivery platform. Angew Chem Int Ed Engl 45: 8149-8152.

58. Hong $H$, Zhang $Y$, Sun J, Cai W (2009) Molecular imaging and therapy of cancer with radiolabeled nanoparticles. Nano Today 4: 399-413.

59. Lupold SE, Hicke BJ, Lin Y, Coffey DS (2002) Identification and characterization of nuclease-stabilized RNA molecules that bind human prostate cancer cells via the prostate-specific membrane antigen. Cancer Res 62: 4029-4033.

60. Farokhzad OC, Cheng J, Teply BA, Sherifi I, Jon S, et al. (2006) Targeted nanoparticle-aptamer bioconjugates for cancer chemotherapy in vivo. Proc Natl Acad Sci U S A 103: 6315-6320.

61. Danhier F, Ansorena E, Silva JM, Coco R, Le Breton A, et al. (2012) PLGA- 
Citation: Ismail SI, Alshaer W, Ababneh N, Fattal E (2013) Aptamers: Promising Molecules for Cancer Stem Cell Targeting. J Mol Genet Med 7: 90 doi: 10.4172/1747-0862.1000090

based nanoparticles: an overview of biomedical applications. J Control Release 161: 505-522.

62. Owens DE 3rd, Peppas NA (2006) Opsonization, biodistribution, and pharmacokinetics of polymeric nanoparticles. Int J Pharm 307: 93-102.

63. Gavrilov K, Saltzman WM (2012) Therapeutic siRNA: principles, challenges, and strategies. Yale J Biol Med 85: 187-200.

64. Nguyen J, Szoka FC (2012) Nucleic acid delivery: the missing pieces of the puzzle? Acc Chem Res 45: 1153-1162.

65. McNamara JO 2nd, Andrechek ER, Wang Y, Viles KD, Rempel RE, et al. (2006) Cell type-specific delivery of siRNAs with aptamer-siRNA chimeras. Nat Biotechnol 24: 1005-1015.

66. Dassie JP, Liu XY, Thomas GS, Whitaker RM, Thiel KW, et al. (2009) Systemic administration of optimized aptamer-siRNA chimeras promotes regression of PSMA-expressing tumors. Nat Biotechnol 27: 839-849.

67. Frei E III (1972) Combination cancer therapy: presidential address. Cancer Res 32: 2593-2607.

68. Kim E, Jung Y, Choi H, Yang J, Suh JS, et al. (2010) Prostate cancer cell death produced by the co-delivery of Bcl-xL shRNA and doxorubicin using an aptamer-conjugated polyplex. Biomaterials 31: 4592-4599.

69. Bates PJ, Laber DA, Miller DM, Thomas SD, Trent JO (2009) Discovery and development of the G-rich oligonucleotide AS1411 as a novel treatment for cancer. Exp Mol Pathol 86: 151-164.

70. Abdelmohsen K, Gorospe M (2012) RNA-binding protein nucleolin in disease. RNA Biol 9: 799-808.

71. Sedletska Y, Giraud-Panis M, Malinge J (2005) Cisplatin is a DNA-damaging antitumour compound triggering multifactorial biochemical responses in cancer cells: importance of apoptotic pathways. Curr Med Chem Anticancer Agents 5: 251-265.

72. Cao Z, Tong R, Mishra A, Xu W, Wong GC, et al. (2009) Reversible cell-specific drug delivery with aptamer-functionalized liposomes. Angew Chem Int Ed Eng 48: 6494-6498

73. Geiger A, Burgstaller P, von der Eltz H, Roeder A, Famulok M (1996) RNA aptamers that bind L-arginine with sub-micromolar dissociation constants and high enantioselectivity. Nucleic Acids Res 24: 1029-1036

74. Jeong S, Han SR, Lee YJ, Kim JH, Lee SW (2010) Identification of RNA aptamer specific to mutant KRAS protein. Oligonucleotides 20: 155-161.

75. Shangguan D, Li Y, Tang Z, Cao ZC, Chen HW, et al. (2006) Aptamers evolved from live cells as effective molecular probes for cancer study. Proc Natl Acad Sci U S A 103: 11838-11843.

76. Daniels DA, Chen H, Hicke BJ, Swiderek KM, Gold L (2003) A tenascin-C aptamer identified by tumor cell SELEX: systematic evolution of ligands by exponential enrichment. Proc Natl Acad Sci U S A 100: 15416-15421.

77. Mallikaratchy P, Tang Z, Kwame S, Meng L, Shangguan D, et al. (2007) Aptamer directly evolved from live cells recognizes membrane bound immunoglobin heavy mu chain in Burkitt's lymphoma cells. Mol Cell Proteomics 6: 2230-2238.

78. Herlyn M, Steplewski Z, Herlyn D, Koprowski H (1979) Colorectal carcinomaspecific antigen: detection by means of monoclonal antibodies. Proc Natl Acad Sci U S A 76: 1438-1442.

79. Marhaba R, Klingbeil P, Nuebel T, Nazarenko I, Buechler MW, et al. (2008) CD44 and EpCAM: cancer-initiating cell markers. Curr Mol Med 8: 784-804.

80. Gires O, Bauerle PA (2010) EpCAM as a target in cancer therapy. J Clin Oncol 28: e239-240.

81. Terris B, Cavard C, Perret C (2010) EpCAM, a new marker for cancer stem cells in hepatocellular carcinoma. J Hepatol 52: 280-281.

82. Went $P$, Vasei $M$, Bubendorf L, Terracciano L, Tornillo L, et al. (2006) Frequent high-level expression of the immunotherapeutic target Ep-CAM in colon, stomach, prostate and lung cancers. Br J Cancer 94: 128-135.

83. Sterzynska K, Kempisty B, Zawierucha P, Zabel M (2012) Analysis of the specificity and selectivity of anti-EpCAM antibodies in breast cancer cell lines. Folia Histochem Cytobiol 50: 534-541.

84. Schmoll HJ, Arnold D (2009) When wishful thinking leads to a misty-eyed appraisal: the story of the adjuvant colon cancer trials with edrecolomab. J Clin Oncol 27: 1926-1929.

85. Shigdar S, Lin J, Yu Y, Pastuovic M, Wei M, et al. (2011) RNA aptamer against a cancer stem cell marker epithelial cell adhesion molecule. Cancer Sci 102 991-998.

86. Screaton GR, Bell MV, Jackson DG, Cornelis FB, Gerth U, et al. (1992 Genomic structure of DNA encoding the lymphocyte homing receptor CD44 reveals at least 12 alternatively spliced exons. Proc Natl Acad Sci U S A 89 : $12160-12164$

87. Afify A, Purnell P, Nguyen L (2009) Role of CD44s and CD44v6 on human breast cancer cell adhesion, migration, and invasion. Exp Mol Pathol 86: 95-100.

88. Choi KY, Saravanakumar G, Park JH, Park K (2012) Hyaluronic acid-based nanocarriers for intracellular targeting: interfacial interactions with proteins in cancer. Colloids Surf B Biointerfaces 99: 82-94.

89. Somasunderam A, Thiviyanathan V, Tanaka T, Li X, Neerathilingam M, et al (2010) Combinatorial selection of DNA thioaptamers targeted to the HA binding domain of human CD44. Biochemistry 49: 9106-9112.

90. Ababneh N, Alshaer W, Allozi O, Mahafzah A, El-Khateeb M, et al. (2013) In vitro Selection of Modified RNA Aptamers Against CD44 Cancer Stem Cell Marker. Nucleic Acid Ther.

91. Griguer CE, Oliva CR, Gobin E, Marcorelles P, Benos DJ, et al. (2008) CD133 is a marker of bioenergetic stress in human glioma. PLoS One 3: e3655.

92. LaBarge MA, Bissell MJ (2008) Is CD133 a marker of metastatic colon cancer stem cells? J Clin Invest 118: 2021-2024.

93. Ferrandina G, Martinelli E, Petrillo M, Prisco MG, Zannoni G, et al. (2009) CD133 antigen expression in ovarian cancer. BMC Cancer 9: 221.

94. Shigdar S, Qiao L, Zhou SF, Xiang D, Wang T, et al. (2013) RNA aptamers targeting cancer stem cell marker CD133. Cancer Lett 330: 84-95.

95. Kim Y, Wu Q, Hamerlik P, Hitomi M, Sloan AE, et al. (2013) Aptamer identification of brain tumor-initiating cells. Cancer Res 73: 4923-4936.

96. Sefah K, Bae KM, Phillips JA, Siemann DW, Su Z, et al. (2013) Cell-based selection provides novel molecular probes for cancer stem cells. Int $\mathrm{J}$ Cancer 132: $2578-2588$.

97. Wang J, Sefah K, Altman MB, Chen T, You M, et al. (2013) Aptamer-conjugated nanorods for targeted photothermal therapy of prostate cancer stem cells. Chem Asian J 8: 2417-2422.

98. Cai Z, Zhang H, Liu J, Berezov A, Murali R, et al. (2010) Targeting erbB receptors. Semin Cell Dev Biol 21: 961-966.

99. Abhold EL, Kiang A, Rahimy E, Kuo SZ, Wang-Rodriguez J, et al. (2012) EGFR kinase promotes acquisition of stem cell-like properties: a potential therapeutic target in head and neck squamous cell carcinoma stem cells. PLoS One 7 : e32459.

100. Perez A, Neskey DM, Wen J, Pereira L, Reategui EP, et al. (2013) CD44 interacts with EGFR and promotes head and neck squamous cell carcinoma initiation and progression. Oral Oncol 49: 306-313.

101. Esposito CL, Passaro D, Longobardo I, Condorelli G, Marotta P, et al. (2011) A neutralizing RNA aptamer against EGFR causes selective apoptotic cell death. PLoS One 6: e24071. 